\title{
Enhanced expression of some biomarkers as a predictive factor for malignant transformation of lesions with atypia in ovarian endometriosis
}

\begin{abstract}
Background: Patients with ovarian endometriosis (OE) are known to have a 2.5 -fold increase in the risk of malignant neoplasms. OE is polymorphic condition histologically subdivided on two forms: "without atypia" and "with atypia". First form is considered benign, while second one is precursor of ovarian malignant transformation.
\end{abstract}

Aim: Assess of the morphological and immunohistochemical characteristics indicative of the risk of malignant neoplastic transformation of $\mathrm{OE}$ atypical lesions.

Materials and Methods: Seventy-eight fragments of ovarian tissue (resected regarding ovarian endometrioid cists) were studied histologically for determination of cystic ovarian endometriosis foci with and without atypia. Thirty-five histological slides with $\mathrm{OE}$ and 8 slides with adenocarcinomas of different types were studied immunohistochemically. Monoclonal antibodies to Ki-67, Bcl-2, p53 proteins and polyclonal antibodies to HNF-1 $\beta$ were used. Results were evaluated by means of Histological score (H-score) method.

Results: 35 foci of cystic OE without atypia and 32 foci with epithelial atypia were observed. In foci with epithelial atypia, the expression of Ki-67, Bcl-2 and HNF-1 $\beta$ was significantly higher relatively to the foci without atypia, while expression of $\mathrm{p} 53$ was the same in foci with and without atypia. From 4 subtypes of studied ovarian adenocarcinomas the expression of HNF-1 $\beta$ was only observed in clear cell adenocarcinoma.

Conclusion: Enhanced expression of biomarkers Ki-67, Bcl-2 and HNF-1 $\beta$ in cystic OE with atypia indicates on the risk of malignant transformation of lesions with atypia. Using of these biomarkers can be useful for differential diagnostics of $\mathrm{OE}$ with and without atypia and for decision about surgical treatment.

Keywords: ovarian endometriosis, endometriosis with atypia, ovarian tissue, epithelial,
Volume 2 Issue 2 - 2018

\author{
Sergey A Levakov, Tatiana A Gromova \\ Department of Obstetrics and Gynecology, First Moscow \\ Medical State University, Russia
}

Correspondence: Sergey A Levakov, Department of Obstetrics and Gynecology, First Moscow Medical State University, 8/2 Trubetskaya Str, Moscow I I 9048, Russian Federation, Tel +7(925)5063 I44, Email levakoff@yandex.ru

Received: April 12, 2018 | Published: April 24, 2018
Abbreviations: OE, ovarian endometriosis; HNF-1 $\beta$, Hepatocyte nuclear factor- $1 \beta$; H-score, Histological score

\section{Introduction}

Endometriosis represents one of the most crucial problems in modern gynecology. It is a multifactorial condition characterized by the pathological growth of tissue resembling the endometrium in its morphological and functional properties, outside the lining of the uterus. ${ }^{1}$ In $2-22 \%$ of cases, endometriosis is diagnosed in the general population following asymptomatic course, in $50-60 \%$ of cases, in patients presenting with pelvic pain, and in $20-30 \%$, in patients with infertility. In postmenopausal women, endometriosis is diagnosed in $2-5 \%$ of cases. ${ }^{2-5}$ The first clinical observations supporting the association between endometriosis and ovarian cancer date back to almost a century ago. ${ }^{6}$ However, it was only in the late 20th century that large-scale epidemiological studies confirmed the 2.5 -fold increase in risk of cancer over a 10 -year follow-up period in women with endometriosis. ${ }^{7}$ Endometriosis does indeed share certain characteristics with malignant conditions, such as the presence of both localized and distant foci, and the propensity for cell migration and invasion resulting in target organ damage. ${ }^{8,9}$ The histogenesis of ovarian endometriosis (OE) and endometriosis-associated tumors remains a matter of dispute. A 2.5-fold increase in the risk of malignant ovarian tumors was shown in OE. ${ }^{10}$ Foci of endometrial epithelial metaplasia are observed in $12.1 \%$ of $\mathrm{OE}$ cases, foci of hyperplasia in $9.4 \%$, foci of atypia in $5.9 \%$, while ovarian cancer is diagnosed in $4.1 \%$ of such cases. ${ }^{11}$ Other studies reported that the prevalence of epithelial atypia foci in OE may reach $35-80 \% .{ }^{12,13}$ Ovarian cancer represents a morphologically and biologically heterogenous group of tumors. Serous tumors are characterized by the mutation in p53 gene, mucinous tumors - by the mutation in K-ras gene, and endomethrioid tumors - by those in beta-katenine and PTEN genes. ${ }^{14}$

The protein $\mathrm{p} 53$ regulates the cell cycle, apoptosis, DNA reparation and suppresses the transcription of the $\mathrm{Bcl}-2$ gene. Mutations in the p53 gene which may occur at any stage of the malignant transformation increase the expression of p53 protein and the risk of various actions of the mutant protein on its "targets" (protein expression is increased in foci of dysplasia as compared to normal tissues). Bcl2 is a mitochondrial oncoprotein, an apoptosis inhibitor, a member of the Bcl-2 (B-cell lymphoma 2 protein) family. The effect of this protein is exerted in the phase of DNA degradation which represents the terminal stage of the process of apoptosis regulation. ${ }^{15} \mathrm{Ki}-67$ is the marker of cell proliferation, which is relatively as informative as the $3 \mathrm{H}$-thymidine label. The expression of $\mathrm{Ki}-67$, which is a nonhistone nuclear protein, allows identify proliferating cells in the late G1, S, M and G2 phases of the cell cycle, with the exception of G0 and early G1 phases, since this protein is degraded within 60 to 90 
minutes after mitosis. A correlation between the level of expression of Ki-67 and the level of mitotic activity of the cells has been clearly demonstrated. ${ }^{16}$ Hepatocyte nuclear factor- $1 \beta(\mathrm{HNF}-1 \beta)$ is a protein of the transcription factors super family which binds to the DNA in the form of a homo- or heterodimer. It has been considered a specific immunohistochemical marker of the clear cell ovarian tumors which are most often associated with OE. Other tumors of the ovaries and the endometrium do not express HNF-1 $1 \beta .{ }^{17-22} \mathrm{HNF}-1 \beta$ plays a crucial role in protecting cells against oxidative stress, suppresses apoptosis by means of interaction with apoptosis inhibitor Bcl-2. The expression of HNF-1 $\beta$ was observed in the epithelium of $40 \%$ of ovarian endometrioid cysts and is more often observed in cases of atypia of the epithelium in endometrioid lesions.

\section{Materials and methods}

In this retrospective study, we included 78 fragments of ovarian tissue retrieved following ovarian resection due to endometrioid cysts, and 8 ovarian adenocarcinomas ( 2 clear cell, 2 welldifferentiated serous, 2 endometrioid and 2 mucinous). Patients with adenocarcinomas had no history of $\mathrm{OE}$ or signs of $\mathrm{OE}$ in retrieved samples. For the histological investigation of the 78 cases, a standard technique was employed using $10 \%$ neutral buffered formalin, paraffin embedding and histological section of $3 \mu \mathrm{m}$ _from paraffin blocks in HM355S rotary microtome (Thermo Scientific, Germany). The sections were further spread on glass slides and hematoxylineosin stained. The assessment of sections allowed confirm and specify the pathohistological diagnosis, to identify endometriotic lesions (apart from the endometriotic cyst which was the reason for surgery) in ovarian tissue, and to select paraffin blocks to perform the immunohistochemical assessment. OE was confirmed using standard morphological criteria, i.e. the presence of epithelial lining of the cyst and/or of glandular components consisting of endometrioid cells (with account of reactive, regeneratory, dystrophic, metaplastic changes, and atypia), the presence of endometrioid stroma and macrophages with hemosiderine contained in the infiltrate and/or cyst lumen. ${ }^{23-25}$ Forty three samples were assessed using the immunohistochemical method - of them, 35 were OE samples (assessed using four mono- and polyclonal antibodies) and 8 samples were ovarian adenocarcinomas: 2 clear cell, 2 well-differentiated serous, 2 endometrioid and 2 mucinous. Patients with adenocarcinomas had no history of OE or signs of OE in retrieved samples.

The four types of adenocarcinomas were assessed for determination of specificity of HNF-1 $\beta$ expression in ovarian tumors. As primary antibodies, we used the antibodies to a cell proliferation marker, nuclear protein Ki-67 (monoclonal antibody, MIB-1 clone, DAKO, Norway, at a dilution of 1:150), apoptosis inhibitor Bcl-2 (monoclonal antibody, clone 124, Cell Marque, USA, at a dilution of 1:250), oncoprotein p53 (monoclonal antibody, clone DO7, Cell Marque, USA, RTU dilution), and transcription factor super family protein, hepatocyte nuclear factor-1beta (HNF-1 $\beta$, polyclonal antibody, GeneTex, USA, at a dilution 1:200). The study was performed using serial $3 \mu \mathrm{m}$ sections produced from paraffin blocks selected in course of preliminary histological assessment of all available tissue specimens from each of the cases. The sections were placed on special covered glass slides for staining (endogenous peroxidase in deparaffinated slides was blocked using 3\% peroxide). Chemical reactions were performed simultaneously for the complete set of specimens in order to obtain comparable data. The sections were deparaffinized on glass with of xylene and further washed with 5 rinses of ethanol in descending concentrations and then with distilled water, following the protocol described in Table 1. We further applied high-temperature antigen retrieval following the technique described by SR.Shi et al. ${ }^{26,27}$ via the incubation of histological sections in a buffer solution (citrate, $\mathrm{pH}$ 6.2, or TRIS-EDTA, $\mathrm{pH} 8.0$, subject to recommendations provided by primary antibody manufacturers) in a RT Module (Thermo Scientific, Great Britain) for heat-induced processing of formalin fixed paraffin-embedded tissue sections. The following settings were selected: temperature $98-99^{\circ} \mathrm{C}$, incubation time $20 \pm 1 \mathrm{~min}$, pre-heating $65^{\circ} \mathrm{C}$.

Table I Histological sections deparaffination protocol

\begin{tabular}{llll}
\hline Rinse № & Solvents & Temperature & Duration (min) \\
\hline I & Xylite I & $20-22^{\circ} \mathrm{C}$ & $10-15 \mathrm{~min}$ \\
2 & Xylite II & $20-22^{\circ} \mathrm{C}$ & $5-10 \mathrm{~min}$ \\
3 & Xylite III & $20-22^{\circ} \mathrm{C}$ & $2 \mathrm{~min}$ \\
4 & Ethanol & $20-22^{\circ} \mathrm{C}$ & $2 \mathrm{~min}$ \\
5 & absolute & $20-22^{\circ} \mathrm{C}$ & $2 \mathrm{~min}$ \\
6 & Ethanol $96 \% \mathrm{I}$ & $20-22^{\circ} \mathrm{C}$ & $2 \mathrm{~min}$ \\
7 & Ethanol $96 \%$ II & $20-22^{\circ} \mathrm{C}$ & $2 \mathrm{~min}$ \\
8 & Ethanol $70 \%$ & $20-22^{\circ} \mathrm{C}$ & $2 \mathrm{~min}$ \\
9 & Ethanol $40 \%$ & $20-22^{\circ} \mathrm{C}$ & $5 \mathrm{~min}$
\end{tabular}

Samples cooled to $85^{\circ} \mathrm{C}$ were transferred on tripods into the unit for immunohistochemical and immunocitological staining, (Autostainer, Thermo Scientific, Great Britain). Immunohistochemical reactions were performed in accordance with the protocols provided by manufacturers for mono- and polyclonal antibodies. For reaction visualisation, we used a ready test-system with universal secondary chromogen (3,3' - diaminobenzydine)-labeled antibodies Histophine (Nichirei Corp., Japan). Two negative controls (for reaction specificity and absence of active endogenous peroxidase) and one internal positive control (for reaction specificity) were accounted for. Results were evaluated by assigning the Histochemical score (H-score) method ${ }^{28,29}$ based on the number and intensity of stained cell nuclei (Ki-67, p53, HNF-1 $\beta$ ) and cytoplasm (Bcl-2): score below 80 was interpreted as low, 80-140 as moderate, and 141-300 as high staining intensity. The evaluation was performed for 100 epithelial cells in three randomly selected fields of view with x400 magnification. The statistical analysis was performed using Statistica for Microsoft 10 software. Mean and the standard deviation were reported for the date sample and a non-parametric Wilcoxon-Mann-Whitney test was performed with a critical value of $0.05(\mathrm{p}<0.05)$.

\section{Results}

Of the 78 assessed cases, in the epithelium of endometrioid cyst walls and/or other endometriotic lesions, the following microfoci or larger foci were observed: with syncytial papillary regenerative (hyperplastic) changes - 31 cases (39.7\%), with hobnail-cell metaplasia in 12 cases $(15.4 \%$ ), with focal hyperplasia (papillary, mixed glandular and solid, with squamous metaplasia or with loci of squamous metaplasia) - 3 cases (3.9\%). In 32 cases (41.0\%), epithelial atypia was identified which in 27 cases (34.6\%) was estimated as "regenerative/dystrophic", ${ }^{23,24}$ due to marked dystrophia of epitheliocytes combined with the accumulation of intraepithelial leucocytes and the inflammatory infiltration of adjacent stroma of 
the endometrioid lesion, while in 5 cases $(6.4 \%)$ such changes were absent and "true neoplastic" atypia was diagnosed. In 1 case $(1.3 \%)$, a small focus of a borderline mucinous tumor was found in the wall of an endometrioid cyst. In 43 of the 78 histologically assessed OE cases $(55.1 \%)$, within the resected ovarian fragments containing an endometrioid cyst, endometriotic lesions (from 1 to 4 ) were identified. Those were presented with epithelial and stromal components both within the cyst walls and at varying distance from it. Summarizes of the results of immunohistochemical assessment of the selected OE and 8 ovarian adenocarcinoma cases are described in the Table 2. As it is seen from the table, in foci with epithelial atypia, the expression level of Ki-67, Bcl-2 and HNF-1 $\beta$ was significantly higher relatively to the foci without atypia. The differences in these marker proteins expression between endometriosis foci with atypia and without atypia are statistically significant $(\mathrm{p}<0.05)$. Expression level of $\mathrm{p} 53$ protein was low in comparison with levels of three other studied proteins and no significant difference in $\mathrm{p} 53$ expression was revealed between $\mathrm{OE}$ foci with and without atypia $(\mathrm{p}>0.05)$.

Table 2 Results of immunohistochemical determination of expression level of biomarkers Ki-67, p-53, BCL-2 and HNF-I $\beta$ in foci of ovarian endometriosis with atypia and without atypia

\begin{tabular}{lll}
\hline Marker protein & $\begin{array}{l}\text { OE foci without atypia } \\
\text { H-score: } \mathbf{M} \pm \boldsymbol{\sigma} \\
\mathbf{n}=35\end{array}$ & $\begin{array}{l}\text { OE foci with atypia } \\
\text { H-score: } \mathbf{M} \pm \boldsymbol{\sigma} \\
\mathbf{n}=32\end{array}$ \\
\hline $\mathrm{Ki}-67$ & $27 \pm 14$ & $79 \pm 11^{*}$ \\
$\mathrm{P} 53$ & $12 \pm 10$ & $16 \pm 12$ \\
$\mathrm{Bcl}-2$ & $43 \pm 11$ & $121 \pm 27^{*}$ \\
$\mathrm{HNF}-\mathrm{IB}$ & $62 \pm 17$ & $188 \pm 13^{*}$
\end{tabular}

Note: $n=35$ is the number of OE foci without of atypia; $n=32$ is the number of $O E$ foci with atypia. The OE foci were selected from 35 ovarian fragments. Each of the fragments contained the foci both with and without atypia, with exception of 3 fragments, which did not contain foci with atypia. *- means that the difference in expression level of the biomarker in foci with atypia compared to foci without atypia is statistically significant $(p<0.05)$

It should be noted, that unlike OE foci with atypia, foci with syncytial papillary regenerative (hyperplastic) changes, with hobnailcell metaplasia, and focal hyperplasia (papillary, mixed glandular and solid, or with loci of squamous metaplasia) did not differ from normal endometrial epithelium in the expression of Ki-67, p53 or Bcl-2. The expression of the specific transcription factor of clear cell ovarian and endometric tumors HNF-1 $\beta$ was observed in epithelial nuclei of the majority of endometrioid lesions. It appeared in the nuclei of both endometrioid cysts epithelium and other endometrioid epithelium lesions (those found in walls of cysts and at a distance from them) regardless of the presence of metaplastic or hyperplastic changes in the epithelium. The evaluation of the number of endometrioid lesions with varying morphological features demonstrated that in OE foci without atypia in $56 \%$ of cases the expression of HNF$1 \beta$ was observed, while in OE foci with atypia (regenerative/ dystrophic or true) HNF-1 $\beta$ was expressed in $94 \%$ of epithelial nuclei. Immunohistochemical assessment of HNF-1 $\beta$ expression in clear cell $(\mathrm{n}=2)$, well-differentiated serous $(\mathrm{n}=2)$, endometrioid $(n=2)$ and mucinous $(n=2)$ adenocarcinomas showed that HNF-1 $\beta$ is indeed enough specific marker for clear cell adenocarcinoma. ${ }^{23}$ Other assessed ovarian tumors actually do not express HNF-1 $\beta$ (only a few single cells with mild intensity of nuclei staining). In the borderline mucinous tumor found in the wall of an endometrioid cyst in one case the expression of HNF-1 $\beta$ was unidentifiable. Expression level of HNF-1 $\beta$ in clear cell appeared significantly higher in comparison with the expression level in OE foci without atypia: $195 \pm 29$ vs $62 \pm 17$ $\mathrm{H}$-score (the difference is statistically significant, $\mathrm{p}<0.05$ ) and was, in fact, equal to the expression level in OE foci with atypia (195 \pm 29 vs $188 \pm 13$ H-score, $\mathrm{p}>0.05$ ).

\section{Conclusion}

The results of this study confirmed the high prevalence of histological, as well as molecular-biological changes in OE lesions suggesting an increased risk of neoplastic transformation. OE lesions with histological signs of epithelial atypia (regenerative/dystrophic in $34.6 \%$ and true neoplastic in $6.4 \%$ ) were characterized by increase expression of the proliferation marker $\mathrm{Ki}-67$, the apoptosis inhibitor Bcl-2 and the specific transcription factor of clear cell ovarian and endometric tumors HNF-1 $\beta$. The observed coincidence of expression levels of HNF-1 $\beta$ in OE lesions with atypia and in clear cell adenocarcinoma, while the both levels are significantly higher of expression level of HNF-1 $\beta$ in OE lesions without atypia (see Table 2), indicates on counteraction of pathogenesis pass ways of $\mathrm{OE}$ lesions with atypia and clear cell ovarian adenocarcinoma. It was reliable established, that $\mathrm{OE}$ most often associated with clear cell adenocarcinoma. Quite probably, that at least part of clear cell adenocarcinomas arise as a result of malignant transformation of OE lesions with atypia. ${ }^{30}$ Using of immunohistochemical markers Ki-67, Bcl-2 and HNF-1 $\beta$, which enhance their expression in OE lesions with atypia may be a useful approach for differential diagnostics of $\mathrm{OE}$ subtypes with and without atypia and preventive surgical treatment of patients with OE.

\section{Acknowledgements}

None.

\section{Conflict of interest}

The authors declare there is no conflict of interest.

\section{References}

1. Clement PB. The pathology of endometriosis: a survey of the many faces of a common disease emphasizing diagnostic pitfalls and unusual and newly appreciated aspects. Adv Anat Pathol. 2007;14(4):241-260.

2. Nezhat F, Datta MS, Hanson V, et al. The relationship of endometriosis and ovarian malignancy: a review. Fertil Steril. 2008;90(5): 1559-1570.

3. Bulletti C, Coccia ME, Battistoni S, et al. Endometriosis and infertility. J Assist Reprod Genet. 2010;27(8):441-447.

4. Kim HS, Kim TH, Chung HH, et al. Risk and prognosis of ovarian cancer in women with endometriosis: a meta-analysis. $\mathrm{Br} J$ Cancer. 2014;110(7):1878-1890.

5. Wilbur MA, Shih IM, Segars JH, et al. Cancer Implications for Patients with Endometriosis. Semin Reprod Med. 2017;35(1):110-116.

6. Sampson JA. Endometrial carcinoma of the ovary, arising in endometrial tissue in that organ. Arch Surg. 1925;10(1):1-72.

7. Ness RB, Cramer DW, Goodman MT. et al. Infertility, fertility drugs, and ovarian cancer: A pooled analysis of case-control studies. $\mathrm{Am} \mathrm{J}$ Epidemiol. 2002;155(3):217-224.

8. Jiang QY, Wu RJ. Growth mechanisms of endometriotic cells in implanted places: A review. Gynecol Endocrinol. 2012;28(7):562-567. 
9. Levakov SA, Gromova TA, Kedrova AG, et al. Endometriosis-associated ovarian cancer. Tumors of female reproductive system. 2016;12(4):4751.

10. Pearce CL, Templeman C. Association between endometriosis and risk of histological subtypes of ovarian cancer: a pooled analysis of casecontrol studies. Lancet Oncol. 2012;13(4):313-385.

11. Prefumo F, Todeschini F. Epitelial abnormalities in cystic ovarian endometriosis. Gynecol Oncol. 2002;84:280-284.

12. Nishida M, Watanabe K, Sato N, et al. Malignant transformation of ovarian endometriosis. Gynecol Obstet Invest. 2000;50(Suppl1):18-25.

13. Ogawa S, Kaku T, Amada S, et al. Ovarian endometriosis associated with ovarian carcinoma: a clinicopathological and immunohistochemical study. Gynecol Oncol. 2000;77(2):298-304.

14. Terasawa K, Toyota M, Sagae S, et al. Epigenetic inactivation of TCF2 in ovarian cancer and various cancer cell lines. Br J Cancer. 2006;94(6):914-921.

15. Tsujimoto Y. Role of Bcl-2 family proteins in apoptosis: apoptosomes or mitochondria? Genes Cells. 1998;3(11):697-707.

16. Scholzen T, Gerdes J. The Ki-67 protein: from the known and the unknown. J Cell Physiol. 2000;182(3):311-322.

17. Amano Y, Mandai M, Yamaguchi K, et al. Metabolic alterations caused by HNF1 $\beta$ expression in ovarian clear cell carcinoma contribute to cell survival. Oncotarget. 2015;126(28):26002-26017.

18. Mandai M, Amano Y, Yamaguchi K, et al. Ovarian clear cell carcinoma meets metabolism; HNF-1 $\beta$ confers survival benefits through the Warburg effect and ROS reduction. Oncotarget. 2015;136(31):3070430714.

19. Suzuki E, Kajita S, Takahashi H, et al. Transcriptional upregulation of HNF-1 $\beta$ by NF- $\kappa$ B in ovarian clear cell carcinoma modulates susceptibility to apoptosis through alteration in bcl-2 expression. $L a b$ Invest. 2015;95(8):962-972.

20. Tsuchiya A, Sakamoto M, Yasuda J, et al. Expression profiling in ovarian clear cell carcinoma: identification of hepatocyte nuclear factor-1 beta as a molecular marker and a possible molecular target for therapy of ovarian clear cell carcinoma. Am J Pathol. 2003;163(6):2503-2512.
21. Kao YC, Lin MC, Lin WC, et al. Utility of hepatocyte nuclear factor$1 \beta$ as a diagnostic marker in ovarian carcinomas with clear cells. Histopathology. 2012;61(5):760-768.

22. Kato N, Sasou S, Motoyama T. Expression of hepatocyte nuclear factor1 beta (HNF-1beta) in clear cell tumors and endometriosis of the ovary. Mod Pathol. 2006;19(1):83-89.

23. Kurman RJ, Ellenson H, Ronnett L. Blaustein's Pathology of the Female Genital Tract. 6th ed. 2011

24. Kurman RJ, Carcanglu ML, Herrington CS, WHO Classification of Tumours of female reproductive Organs. 4th ed. Lyon: WHO Press; 2014.

25. Zaino R, Carinelli SG, Ellenson LH. Tumours of the uterine corpus: epithelial tumours and precursors. Lyon: WHO Press; 2014:125-126.

26. Shi SR, Imam SA, Young L, et al. Antigen retrieval immunohistochemistry under the influence of $\mathrm{pH}$ using monoclonal antibodies. Journal Histochem Cytochem. 1995;43(2):193-201.

27. Shi SR, Chaiwun B, Young L, et al. Antigen retrieval technique utilizing citrate buffer or urea solution for immunohistochemical demonstration of androgen receptor in formalin-fixed paraffin sections. Journal Histochem. Cytochem. 1993;41(11):1599-1604.

28. Cohen DA, Dabbs DJ, Cooper KL, et al. Interobserver Agreement Among Pathologists for Semiquantitative Hormone Receptor Scoring in Breast Carcinoma. Am J Clin Pathol. 2012;138(6):796-802.

29. McCarty KS, Miller LS, Cox EB, et al. Estrogen receptor analyses. Correlation of biochemical and immunohistochemical methods using monoclonal antireceptor antibodies. Arch Pathol Lab Med. 1985;109(8):716-721.

30. Sainz de la Cuesta R, Eichhorn JH, Rice LW, et al. Histologic transformation of benign endometriosis to early epithelial ovarian cancer. Gynecol Oncol. 1996;60(2):238-244. 